\title{
Análisis de la satisfacción del turista con relación a factores tecnológicos y la autogestión de la información
}

\author{
Cecilia Vélez ${ }^{(1)}$, Oscar J. Alejo(2), Guillermo Tafur ${ }^{(1)}$ y Miguel A. Bustamante(1,3)* \\ (1) Facultad de Ciencias Económicas y Administrativas, Universidad Católica de Santiago de Guayaquil. Av. Carlos Julio \\ Arosemena Km. 11/2 vía Daule, Guayaquil, Ecuador (correo-e: cecilia.velez@cu.ucsg.edu.ec, \\ guillermo.tafur@cu.ucsg.edu.ec) \\ (2) Instituto Superior Universitario Bolivariano de Tecnología. Víctor Manuel Rendón 236 y Pedro Carbo, Guayaquil, \\ Ecuador (correo-e: oalejo@bolivariano.edu.ec) \\ (3) Facultad de Economía y Negocios, Universidad de Talca, 2 norte 685, Talca, Chile. (correo-e: mabu@utalca.cl)
}

${ }^{*}$ Autor a quien debe ser dirigida la correspondencia.

Recibido Dic. 2, 2019; Aceptado Ene. 28, 2020; Versión final Mar. 23, 2020, Publicado Ago. 2020

\begin{abstract}
Resumen
El presente trabajo determina el nivel de satisfacción percibido por los turistas a la incorporación de las tecnologías de la información y las comunicaciones en la difusión y gestión de los procesos del sector turismo. Se desarrolló un estudio no experimental y descriptivo de diseño transversal con enfoque cuantitativo mediante el análisis de las innovaciones informáticas introducidas al sector turismo y las percepciones asociadas de los visitantes. Se observa un incremento de las tasas de llegadas internacionales y de gasto turístico, lo que contribuye al nivel de inversión en turismo. Esto se correlaciona positivamente con el empleo e incrementa el crecimiento sectorial del turismo en la economía de Guayas. Se concluye que la percepción de los turistas es de alta satisfacción y que las redes sociales y aplicaciones móviles siguen ganando adeptos, siendo más frecuentes las plataformas de Booking y Facebook y en el territorio del Guayas, los Kioscos digitales.
\end{abstract}

\section{Analysis of tourist satisfaction in relation to technological factors and self-management of information}

\begin{abstract}
The present study determines the level of satisfaction perceived by tourists with the incorporation of information and communication technologies in the dissemination and management of processes in the touristic sector. A non-experimental and descriptive study of cross-sectional design with a quantitative approach is developed by analyzing the computer innovations introduced to the tourism sector and the associated perceptions of visitors. The results show an increase in the rates of international arrivals and tourist spending, which contributes to the level of tourism investment. This correlates positively with employment levels and with increases in growth of the tourism sector in the Guayas economy. It is concluded that the perception of tourists is of high satisfaction and that social networks and mobile applications continue to gain support. The most frequent interaction mechanisms are the Booking and Facebook platforms and in the territory of Guayas the digital Kiosks.
\end{abstract}




\section{INTRODUCCIÓN}

El turismo es una actividad que incide directamente en los ámbitos económico, social y cultural de un país proveyendo beneficios significativos para las economías, especialmente aquellas de nivel medio, que repercute progresivamente en su desarrollo (Díaz y Onofri, 2017), sin embargo, el crecimiento sectorial puede generar efectos adversos sobre los ecosistemas, los que aunque pasen inadvertidos, con el tiempo son visibles y se pueden observar en el uso de recursos por parte de los proveedores de servicios turísticos (Llorente-Bousquets y Ocegueda, 2008). Es por ello que se lo define como uno de los ejes dinamizadores de la economía a escala mundial, en tanto propicia el progreso a través de la participación de los sectores público, privado y comunitario (Mendoza, et al., 2016).

Para algunos analistas sectoriales, es un fenómeno social y económico que incide sobre diversos grupos sociales (Herington, et al., 2013), es apreciado positivamente por las organizaciones gubernamentales y no gubernamentales y, en general por todas las comunidades, que conforman los destinos turísticos (Song, et al., 2012), consecuencia de lo cual, es preciso relevar la importancia de variables no económicas, de carácter social y de corte cualitativo, como son las percepciones que motivan e influyen en la decisión final del visitante para acceder a un destino turístico (Sung, et al., 2015).

El turismo es un importante medio generador de ingreso de divisas para un país (Díaz y Onofri, 2017) y representa una fuente de trabajo y capital de gran magnitud en tanto aporta al producto interno bruto (PIB) de los países. El turismo, es entonces, uno de los renglones más importantes del sector terciario de la actividad económica global (OMT, 2016), representa el 10\% del PIB mundial, el 7\% del comercio internacional y el 30\% de las exportaciones de servicios. Complementariamente, el turismo es en sí mismo un hecho social que influye en la identidad de los países, permite su crecimiento y fortalece su identidad (Webster y lvanov, 2014) y, por ello mismo, estimula la cooperación público-privada y, a partir de ello, contribuye a reducir los índices de pobreza del país receptor (Díaz y Onofri, 2017).

Ecuador, considerado uno de los 12 países mega diversos del mundo, presenta una actividad turística multicultural (Assaker, et al., 2011; Llorente-Bousquets y Ocegueda, 2008) que está experimentando cambios profundos incorporando nuevas tecnologías de la información y las comunicaciones, por una parte, para sentar las bases de un turismo sostenible (Alegre y Garau, 2011) así como para generar respuestas innovadoras que satisfagan con calidad a los turistas (Gracia y Torres, 2015; Reyes et al., 2015; Webster y Ivanov, 2014). Es por ello que el sector en su conjunto, vía las acciones de los organismos públicos y entidades privadas, ha incorporado las tecnologías de la información y las comunicaciones en sus diversas opciones tecnológicas (Tafur et al., 2018), las cuales este trabajo busca analizar a través del estudio de las percepciones de los turistas nacionales e internacionales que visitan el Guayas.

La percepción es el proceso complejo de detección y atención por medio del cual las personas seleccionan, organizan e interpretan una realidad que les provoca sensaciones, dándoles un significado que es capaz de gatillar una conducta (Solomon, 2008). Sin perjuicio de ello se identifican estereotipos auto definidos acerca de cómo debe expresarse la realidad, que puede pasar inadvertida porque su percepción es subliminal o bien consciente si se activa una alerta perceptual efectiva en contraposición a la defensa perceptual según la cual la gente ve lo que desea y no advierte lo que no quiere ver produciendo una ilusión perceptual (Gregory, 2005). Sin embargo, a través de la adaptación, las personas se percatan, atienden y se habitúan a un hecho que se les hace trivial razón por la cual, las innovaciones, en este trabajo, provenientes de las tecnologías de la información y las comunicaciones dan pie a esperar que, en este caso los turistas, se comporten de acuerdo con un posicionamiento perceptual (Solomon, 2008) que les permita percatarse de las características de lo que se les ofrece en términos de los medios de contacto y acceso tecnológico a las ofertas de turismo del Guayas.

Sobre la base de lo señalado, el sector turismo de Ecuador ha incorporado activamente los avances provenientes de las tecnologías de la información y las comunicaciones en sus procesos de difusión y gestión (CGTI, 2018). Estas acciones han complejizado progresivamente los procesos en la medida que éstos proporcionan nuevas capacidades de la mano de las nuevas tecnologías que entregan, a la autonomía de los usuarios, el acceso y uso de los servicios de turismo, por ejemplo mediante accesos en línea y de autogestión tecnológica, proveyéndole a las personas de nuevas capacidades de respuesta y de servicios amigables y accesibles (Castillo, et al., 2016; Gracia y Torres, 2015; Sung, et al., 2015) que reportan satisfacción, a causa de la autogestión de los medios y el uso autónomo de las tecnologías de la información por parte de los turistas, en tanto les permita identificar, seleccionar, reservar y comprar los servicios requeridos (Song, et al., 2012). Sobre la base de los conceptos analizados y considerando la necesidad de conocer la percepción de los turistas (Fernández y Cuadrado, 2014), el presente trabajo busca determinar el nivel de satisfacción percibido por parte de los turistas nacionales e internacionales a la incorporación de las tecnologías de la información y las comunicaciones a los diversos canales de difusión y gestión del sector turístico del Guayas, Ecuador. 


\section{METODOLOGÍA}

El presente trabajo, de carácter no experimental, descriptivo y de diseño transversal (Hernández et al., 2014) se ejecuta mediante un enfoque cuantitativo que se implementa mediante el método de encuesta a través de un cuestionario debidamente estructurado y cuyos datos fueron tratados, en forma descriptiva lo que incluyó la determinación de frecuencias absolutas y relativas, además de realizar una adecuada descripción de la muestra. La población objeto de estudio fue de turistas nacionales e internacionales que visitan Guayaquil determinada sobre la base de los datos del boletín oficial turismo (CGTI, 2018). Además, dado que el número de registros determinado sobrepasa las 100.000 personas, se procedió a aplicar un método de muestreo.

La muestra determinada a priori previó una varianza máxima $\sigma^{2}=0,25$ considerando una distribución normal de la población. Se contempló un intervalo de confianza $\mu=95 \%$ ( 1 - b probabilidad de error) equivalente a una longitud aproximada $Z=1,96$ y se consideró un error de estimación $\mathrm{e}=5 \%$ como el máximo dispuesto a admitir para el estudio (Freiberg, et al., 2013; Hernández et al., 2014), alcanzándose una muestra representativa estimada $\eta=384$ unidades. El plan de muestreo incluyó la selección aleatoria de turistas nacionales e internacionales que visitan el Guayas.

\section{Instrumento de investigación, confiabilidad y validez}

El cuestionario aplicado incluye las variables integración tecnológica, centralización y autogestión de información turística (Alegre y Garau, 2011; Gracia y Torres, 2015), además de los ítems demográficos de generación, nacionalidad, lugar de preferencia y frecuencia de visitas (Freiberg, et al., 2013; Hernández et al., 2014). Se verificó la validez de contenido del instrumento y de los reactivos, sobre la base de contrastar con la teoría y la de constructo, se logró a través de grupos focales para ratificar la definición operativa de las variables (Freiberg, et al., 2013). La validez convergente, se logró mediante el análisis de factores de componentes principales, con previa verificación de pertinencia del índice KMO y prueba de esfericidad de Bartlett (Hernández et al., 2014), considerando un índice KMO y esfericidad ( $\left.{ }^{* * *} p=0,001\right)$ significativos. Finalmente, la validez discriminante se confirmó por las varianzas explicadas de los factores y las cargas de los componentes con cada factor. Para garantizar la validez convergente se optó por validar cargas factoriales significativas $C \geq 0,5$ (Freiberg, et al., 2013).

En el proceso de validación se recurrió a consultar a 10 profesionales expertos del sector turístico que incorporan en sus desempeños las tecnologías de la información y las comunicaciones, destacando entre éstos, gerentes generales, expertos con grado de magister en hotelería y turismo, investigadores y planificadores estratégicos en temas de turismo y expertos en tecnologías vinculados al sector turístico quienes verificaron lenguaje, uni-direccionalidad, estructura semántica, consistencia y validez de las taxonomías utilizadas. Recogidas las sugerencias de los expertos, se introdujeron las correcciones y adecuaciones de texto, semántica, contenido y diseño hasta obtener el cuestionario definitivo (Hernández et al., 2014). Finalmente, se realizó el pre-testeo de campo del cuestionario para chequear comprensión, lenguaje, formato, instrucciones y escalas de respuesta, y descartar dudas respecto de las preguntas. El piloteo se realizó con una muestra aleatoria de 50 sujetos (Hernández et al., 2014) que incluyó turistas y visitantes verificando que no fuesen incorporados en la muestra del estudio general. Esta medición permitió, además, verificar la fiabilidad de las escalas (Freiberg, et al., 2013) aplicándose a 20 turistas nacionales y 30 internacionales.

\section{Trabajo de campo y procesamiento de datos}

El levantamiento de la información, por el método de encuestas (Hernández et al., 2014), se llevó a cabo en el mes de mayo del año 2018 en las ubicaciones con mayor concurrencia de Guayaquil, lo que contempló el Aeropuerto José Joaquín de Olmedo de Guayaquil, Terminal Terrestre, Malecón 2000 - Simón Bolívar, Unicentro, Parque Seminario, también conocido como Parque Bolívar o Parque de las Iguanas, Centro Bahía, Malecón del Salado y el Parque Histórico de Guayaquil, en los horarios de 9H00 a 13H00, y de 17H00 a $21 \mathrm{H} 00$ (Freiberg, et al., 2013).

Dada la naturaleza de las preguntas, se realizó un análisis descriptivo de la muestra (Freiberg, et al., 2013) mediante el sistema de procesamiento que proporciona el programa SPSS, V20. Para cada una de las variables se determinaron las frecuencias de percepciones y a partir de las escalas utilizadas, se aplicaron comparaciones de promedios de los sujetos respecto al punto central de la escala. La confiabilidad se verificó mediante el Alfa ( $\alpha$ ) de Cronbach que mide la consistencia interna de los ítems analizados (Hernández et al., 2014) y se optó por valores recomendados (Freiberg, et al., 2013), verificando que el índice de fiabilidad compuesta fuese superior al valor recomendado $(\alpha \geq 0,7)$ (Freiberg, et al., 2013) y la varianza extraída máxima $\sigma^{2} \geq 0,25$ (Alegre y Garau, 2011). 


\section{RESULTADOS}

A continuación, se presentan los resultados comenzando con los principales mercados turísticos, el estado de avance del turismo, así como la percepción de los turistas nacionales e internacionales usuarios de las tecnologías orientadas al turismo en el Guayas.

\section{Principales mercados turísticos con destino a Ecuador}

En la Tabla 1 se visualiza un ranking de llegadas de turistas de acuerdo con los registros de la Coordinación General de Estadística e Investigación (CGTI, 2018).

Ecuador, al ser considerado uno de los países con más diversidad natural del mundo (Llorente-Bousquets y Ocegueda, 2008), se constituye en un mercado turístico de nivel mundial. Como se aprecia en la Tabla 1, el lugar de procedencia de los turistas permite afirmar que, en su mayoría, provienen de América del Sur, incluyendo un $62 \%$ de turistas nacionales. Complementariamente, del análisis proporcional de turistas nacionales e internacionales se determinó que el 73,7\% de estos han visitado en más de 5 ocasiones los atractivos turísticos de la provincia.

Desde el año 2015 el número de turistas nacionales que visitan el Guayas asciende aproximadamente a 1.740 .000 , lo cual indica que como promedio y sin considerar temporadas claves, el ingreso mensual de turistas alcanza 145.000 ecuatorianos que visitan Guayaquil (Mendoza et al., 2016). Así mismo, del anuario de entradas y salidas internacionales y nacionales, se pudo determinar un promedio de $30 \%$ de los extranjeros que llegan a Ecuador visitan en particular la ciudad de Guayaquil.

Durante el año 2017, el turismo se ubicó en la tercera posición de las exportaciones no petroleras (Castillo, et al., 2016). El ingreso de divisas por Turismo alcanzó los USD 1.204,5 millones, representando un crecimiento del 12,0\% respecto al 2016 lo que arrojó una balanza turística con saldo positivo de USD 374,2 millones. Además, al analizar las cifras a nivel de continente se observa que los turistas europeos son quienes más destinan dinero para sus gastos de viaje cuando visitan Ecuador (ENEDyS, 2017).

\begin{tabular}{|c|c|c|c|c|}
\hline \multirow{2}{*}{ País } & \multicolumn{2}{|c|}{ ENERO 2017} & \multicolumn{2}{|r|}{ ENERO 2018} \\
\hline & Registros & $\%$ & Registros & $\%$ \\
\hline Venezuela & 14.901 & $9,80 \%$ & 62.506 & $31,70 \%$ \\
\hline Colombia & 42.232 & $27,90 \%$ & 43.241 & $21,90 \%$ \\
\hline Estados Unidos & 19.575 & $12,90 \%$ & 17.949 & $9,10 \%$ \\
\hline Perú & 15.704 & $10,40 \%$ & 16.064 & $8,10 \%$ \\
\hline Argentina & 7.312 & $4,80 \%$ & 6.715 & $3,40 \%$ \\
\hline Chile & 5.871 & $3,90 \%$ & 5.417 & $2,70 \%$ \\
\hline España & 4.737 & $3,10 \%$ & 4.770 & $2,40 \%$ \\
\hline Canadá & 3.313 & $2,20 \%$ & 3.174 & $1,60 \%$ \\
\hline Cuba & 3.054 & $2,00 \%$ & 2.827 & $1,40 \%$ \\
\hline Alemania & 3.550 & $2,30 \%$ & 2.677 & $1,40 \%$ \\
\hline Otros países & 31.181 & $20,60 \%$ & 31.879 & $16,20 \%$ \\
\hline Total general & 151.430 & $100 \%$ & 197.219 & $100 \%$ \\
\hline \multicolumn{5}{|c|}{ Proyecciones de variables turísticas en Ecuador (2015 - 2025) } \\
\hline Indicadores turísticos & \multicolumn{2}{|c|}{2015} & 2025 & Tasa anual de crecimiento \% \\
\hline Llegada de turistas internacionales & \multicolumn{2}{|c|}{1506} & 2346 & 4,5 \\
\hline Gasto turístico (USD Millones) & \multicolumn{2}{|c|}{1395} & 2146 & 4,3 \\
\hline Inversiones en turismo (USD millones) & \multicolumn{2}{|c|}{1078} & 1658 & 4,3 \\
\hline Empleo (miles) & \multicolumn{2}{|c|}{373} & 574 & 4,3 \\
\hline Contribución al PIB (\%) & \multicolumn{2}{|c|}{5,5} & 8,5 & 4,4 \\
\hline
\end{tabular}

Sin perjuicio de lo indicado, es menester indicar que la cifra de turistas provenientes de Venezuela registrados al año 2018, presenta datos alterados por cuanto las cifras habituales de turistas se vieron incrementadas por tres veces, a causa de la emigración obligada de familias de venezolanos a causa del grado de inestabilidad de su país producto de otras causas coetáneas, como es la caída de los precios del petróleo que afecta duramente a la economía de ese país. 
Por otra parte, desde la perspectiva económica, el empleo en Ecuador recibió los impactos positivos del turismo. Desde el año 2014 al 2017, el turismo aportó con nuevas plazas de trabajo equivalentes al 5,2\% y $6,1 \%$ de los empleos del país, considerando establecimientos tales como restaurantes, alojamientos, entretenimientos entre otros, incrementando las oportunidades de las actividad adicionales al turismo (ENEDEyS, 2017). Complementariamente, algunas cifras tomadas de Consejo Mundial de Viajes y Turismo (CMVyT, 2015), resultan concordantes entre las tasas de crecimiento de las llegadas internacionales $(4,4 \%)$ y el nivel de gasto turístico que se ubica en una proporción similar $(4,2 \%)$ idéntico al nivel de la inversión en turismo que se incrementa en un porcentaje equivalente $(4,2 \%)$.

Por último, las cifras de la proyección al año 2025, se correlacionan con el empleo, el cual como se dijo, incrementó las tasas de ocupación entre 2014 y 2017 lo que se traduce en una contribución neta sectorial del turismo a la economía ecuatoriana que incrementa los flujos hacia el país $(4,2 \%)$ y que se manifiesta proyectada al año 2025, en un 4,4\% de contribución al PIB, como se muera en la tabla 1.

\section{Confiabilidad y validación de las dimensiones del instrumento}

La confiabilidad del instrumento se determinó a través del índice $\alpha$ de Cronbach el cual, para la totalidad de las variables (42) alcanzó un valor $\alpha=0,988$ con una significación alta ( $\left.{ }^{\star \star \star} p=000\right)$, permitiendo que el cuestionario sea calificado de excelente.

Para medir la validez del instrumento, se utilizó el análisis factorial mediante el cual se pudo determinar la medida de adecuación muestral de Kaiser-Meyer-Olkin que alcanzó un índice $\mathrm{KMO}=0,971$, con un chicuadrado aproximado de 35614,731 y una prueba de esfericidad de Bartlett de 861 con una significancia alta ${ }^{* * *} p=000$ ), todo lo cual demuestra que el instrumento se comporta adecuadamente para el análisis dimensional relevante de presente estudio.

En general, considerando la totalidad de los ítems analizados, se determinaron cuatro factores independientes que se comportan de manera autónoma y que confirma la valides discriminante de estos constructos. A su vez, cada factor tiene la capacidad de agrupar ítems correlacionados, confirmando por esta vía la validez convergente de cada uno de éstos que se ratifica mediante los índices de comunalidad. Los factores determinados alcanzan una varianza total explicada de $87,34 \%$, considerada muy buena.

Adicionalmente, se pudo determinar que la totalidad de las preguntas se comportan en forma adecuada alcanzando cargas factoriales altas $(0,966-0,513)$ por encima de los mínimos requeridos para el estudio, así como índices de comunalidad también elevados $(0,993-0,464)$ también superiores a los niveles mínimos estándares para confirmar la contribución de cada reactivo al estudio. Finalmente, se confirma que las preguntas del cuestionario se agrupan de manera pertinente y de una forma característica, facilitando una recogida de datos pertinentes que permitan interpretar las percepciones de los sujetos entrevistados.

En general, los datos de la confiabilidad como de la validación, permiten ratificar que las variables del análisis cumplen el papel requerido en la recogida de datos, son independientes entre sí y no existe, a priori, una dependencia conceptual de unas variables sobre otras, en consecuencia, el instrumento permite definir grupos homogéneos de variables que se correlacionan adecuadamente entre sí y permiten confirmar que los ítems se comportan adecuadamente.

\section{Análisis descriptivo del turísmo hacia el Guayas}

La tabla 2 muestra una síntesis descriptiva de las dimensiones de análisis de la muestra categorizada por tipos de visitantes (ecuatorianos y extranjeros).

El muestreo simple permitió determinar un tamaño de muestra que alcanza $\eta=384$ casos que cuenta con un $54,4 \%$ de mujeres (209) y $45,6 \%$ de hombres (175). Se determinó que el $81 \%$ de los turistas que visitan la Provincia del Guayas tienen edades entre 19 a 49 años, lo que incluye la denominada Generación $Y$ tambien identificada como Millennials (45\%), seguido de la Generación X (36\%) mayormente extranjeros y las generaciones Baby Boomers y $Z$ que marcan frecuencias discretas de $10 \%$ y $9 \%$, respectivamente, mayormente ecuatorianos. Los turistas provienen principalmente de américa del sur ( $80 \%$ ) además de un $8 \%$ que proviene de América del norte.

Por otra parte, respecto del número de veces que los turistas han visitado Ecuador, en su mayoría han concurrido entre 3 y 5 veces (31\%) seguido de quienes han visitado 1 y 2 veces (25\%), en ambos casos con alta presencia de extranjeros y más de 10 veces alcanzando también una frecuencia de visitas del $25 \%$, pero en este caso con predominio de los turistas ecuatorianos. Los resultados observados sugieren además una fuerte orientación turística orientada hacia el turismo de experiencia $41 \%$, principalmenmtye de turistas 
ecuatorianos, seguido del turismo de negocios $32 \%$ con alta freciencia de extranjeros y el turismo cultural $11 \%$ y terminando con el turismo ecológico que llega a un $5 \%$, en estos dos últimos casos en su mayoría de visitantes ecuatorianos.

\begin{tabular}{|c|c|c|c|c|c|}
\hline \multicolumn{6}{|c|}{ Tabla 2: Frecuencias relativas por dimensiones de análisis (\%) } \\
\hline Dimensiones & Categorías & Ecuatorianos & Extranjeros & $\%$ & Total \% \\
\hline \multirow{2}{*}{ Genero } & Hombres & 51,9 & 48,1 & 45,6 & \\
\hline & Mujeres & 50,3 & 49,7 & 54,4 & 100 \\
\hline \multirow{4}{*}{ Generación } & Baby Boomers & 54,5 & 45,5 & 10 & \\
\hline & Generación X & 44,0 & 56,0 & 36 & \\
\hline & Generación Y & 50,3 & 49,7 & 45 & \\
\hline & Generación Z & 80,0 & 20,0 & 9 & 100 \\
\hline \multirow{5}{*}{ Lugar de procedencia } & Asia & 0,0 & 100,0 & 4 & \\
\hline & Europa & 10,0 & 90,0 & 6 & \\
\hline & América del sur & 62,2 & 37,8 & 80 & \\
\hline & 2 América central & 14,3 & 85,7 & 2 & \\
\hline & 1 América del norte & 3,7 & 96,3 & 8 & 100 \\
\hline \multirow{4}{*}{$\begin{array}{l}\text { Número de veces que } \\
\text { visita el Guayas }\end{array}$} & Más 10 veces & 70,6 & 29,4 & 25 & \\
\hline & 6 a 10 veces & 59,7 & 40,3 & 18 & \\
\hline & 3 a 5 veces & 37,7 & 62,3 & 31 & \\
\hline & 1 a 2 veces & 42,2 & 57,8 & 25 & 100 \\
\hline \multirow{5}{*}{ Tipos de turismo } & Turismo de experiencia & 54,7 & 45,3 & 41 & \\
\hline & Turismo de negocios & 23,5 & 76,5 & 32 & \\
\hline & Turismo cultural & 58,3 & 41,7 & 11 & \\
\hline & Turismo ecológico & 63,6 & 36,4 & 5 & \\
\hline & Otros & 53,2 & 46,8 & 11 & 100 \\
\hline
\end{tabular}

\section{Mecanismos de contacto de turismo y tecnología en el Guayas}

Del análisis de la figura 1, que muestra las frecuencias totales relativas del uso de la tecnología, se puede apreciar que, a pesar de que el uso de las aplicaciones móviles (App) sigue ganando adeptos (13\%) la mayoría de los turistas entrevistados continúan utilizando como opción primaria el ir directamente a las agencias de viajes (32\%), así como realizando compras de paquetes por las redes sociales (22\%) o contactar oficinas de turismo (19\%). Finalmente, sólo el $8 \%$ de los turistas encuestados gestionó sus viajes utilizando plataformas web. Sin perjuicio de la primacía de las agencias de viaje como entidad de contacto, las frecuencias relativas indican que se está dando un cambio paulatino de los modelos de negocio tendiendo hacia las tecnologías de las comunicaciones y la información (Webster e Ivanov, 2014).
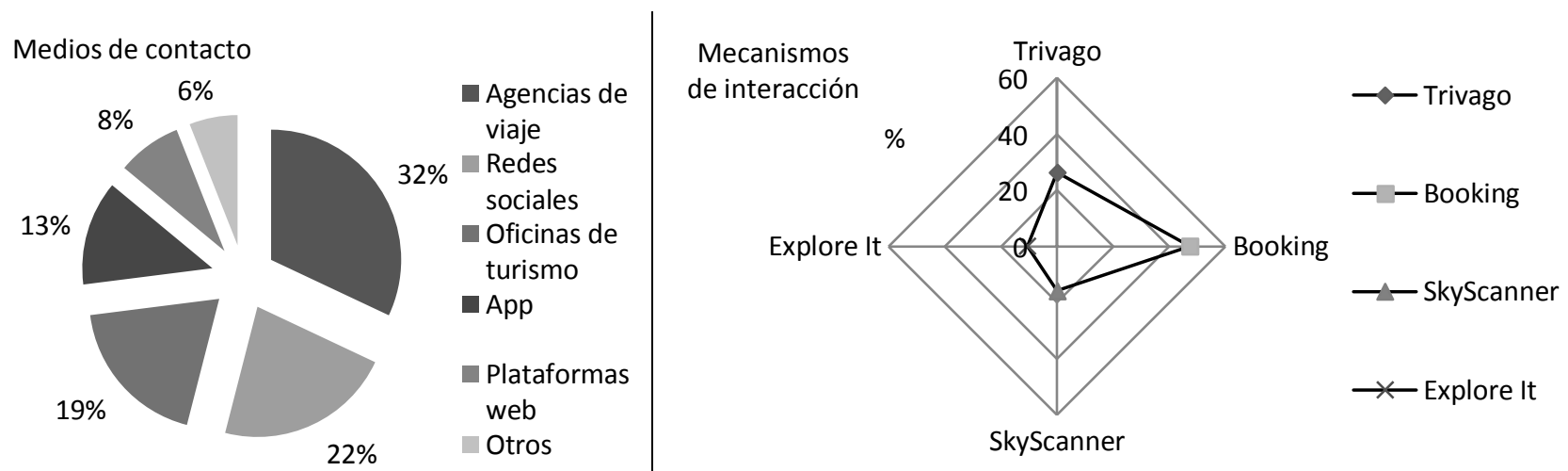

Fig. 1: Medios de contacto e interacción de los turistas que visitan el Guayas

En este contexto, el año 2016 salió al mercado una aplicación móvil bajo la campaña All You Need is Ecuador (todo lo que necesitas es Ecuador) denominada Discover Ecuador \& More (descubre Ecuador y más), esperando se convierta en la principal aplicación turística y comercial de Ecuador (Castillo, et al., 2016). Por otro lado, para la promoción específica de la ciudad de Guayaquil se desarrolló mediante la página web denominada Guayaquil es mi destino en la cual se han ido incorporando diversas estrategias del turismo 2.0 para garantizar la presencia de marca, navegabilidad del sitio y su interactividad (Tafur et al., 2018). Adicionalmente en este contexto, se creó una aplicación móvil con el mismo nombre para acceder a tales servicios a través de teléfonos inteligentes y tablets que generan satisfacción y mejoran la imagen de un destinoi turístico (Assaker, et al., 2011). Se observa así mismo, que la cuenta en Facebook Guayaquil es mi destino ha llegado a lograr aproximadamente 342.039 seguidores, número que supera en un $95,45 \%$ a los 
seguidores en 2016. De estos seguidores, un 95,7\% son ecuatorianos (Larrea y Cevallos, 2016) lo cual indica que el impacto de esta estrategia ha sido a escala local.

En cuanto a las suscripciones en el canal Youtube, Guayaquil es mi destino, ha llegado a totalizar 1.214 seguidores, lo que representa un registro superioir a los subscritos en octubre de 2016 (Tafur et al., 2018). En Twitter se observa además un incremento de un $97,17 \%$ en relación al número que tenian hace dos años atrás. Estas cifras resultan individualmente relevantes aunque sin embargo, juntas demuestran la efectividad de los esfuerzos de difusión de Guayaquil como destino turístico (Tafur et al., 2018). Por otro lado, la plataforma digital para el turismo denominada Goraymi.com, en desarrollo desde el año 2015 se ha convertido en un proyecto consolidado, dando pie a una plataforma digital que impulsa al turismo local desde al año 2017 (Tafur et al., 2018), lo que se confirma por llegar a ser la "start up" ecuatoriana que logró ser reconocida y apreciada a escala mundial (Fernández y Cuadrado, 2014).

Complementando el análisis, según la recopilación de Rivera y Símbala (2016), el sitio web Guayaquil es mi Destino ocupaba en ese año (2016) la posición 2.144 como página más visitada en Ecuador, ubicación que, de acuerdo con el presente estudio, ha mejorado considerablemente a febrero de 2019 aumentando 27 puestos a nivel de Ecuador lo cual mejora su presencia también a nivel internacional y que puede ser considerado un salto considerable que evidencia, por si sólo, la atractividad de este sitio web en la industria turistica. Sin perjuicio de lo indicado, aún queda bastante por hacer en tecnología turística local. Por ejemplo, en torno a las innovaciones tales como cerraduras digitales, granizadoras, jugueras, pantallas táctiles y software administrativo, hasta aplicaciones y sistemas con inteligencia artificial que creen ambientes frescos y novedosos de aplicación en cadenas hoteleras nacionales que dispongan de cierto respaldo tecnológico (Fernández y Cuadrado, 2014). Al respecto, una recomendación de política pública sugerida por las cifras de la Superintendencia de Bancos del Ecuador, busca impulsar un aumento de la colocación de créditos hacia este sector desde la banca pública debido a que este sector es considerado prioritario (PLANDETUR, 2020; SNPyD, 2013).

En términos territoriales, la venta de destinos hacia Ecuador parece evidente en sitios tales como Galápagos, Otavalo, Quito, y Amazonia (Tafur et al., 2018), destinos que los operadores turísticos foráneos decían desconocer (Croes et al., 2009). Por otro lado, el futuro para los operadores turísticos está en la oportunidad que se les presenta para posicionarse como tiendas virtuales del tipo App Stores (13\%) como lo muestra la figura 2, puesto que mejora la oferta de los prestadores y amplia el acceso a los turistas, permitiendo que el destino del Guayas se potencie, mejore los servicios de viajes la disponibilidad de las tecnologías y la seguridad para los viajeros (World Travel Market, 2016), lo que resulta aún escasamente apreciado como páginas Web $(8 \%)$ y mayormente valorado en Redes sociales $(22 \%)$ en un escenario que se está reorganizando (CMVyT, 2015), por una parte para retener clientes, aumentar el uso profesional de bases de datos y por otra, maximizar finalmente la rentabilidad de los prestadores (Webster e Ivanov, 2014).

En síntesis, la plataforma Booking es una de las más referidas (47\%), seguido de Trivago (26\%) consideradas las de mayor frecuencia de uso. Entre las menos utilizadas se mencionan SkyScanner (16\%) y Explorer It (11\%), como se aprecia en la Figura 2. A la fecha de la entrega de esta investigación, estaban en desarrollo más de 300 aplicaciones en línea que tendrían, de acuerdo con la teoría disponible, beneficios diversos tales como reducción de costos en gastos de hospedaje, incremento de las ventas, mejora de la productividad, aumento de las oportunidades de negocio, así como la capacidad de generación de nuevos empleos (Webster e Ivanov, 2014).

\section{Preferencias de contacto y comunicación}

Los nuevos paradigmas de desarrollo tecnológico plantean las ventajas de la centralización, autogestión y auto consulta de la información por parte de los clientes. En este sentido, el estudio determinó que el 92,8\% de los turistas que colaboraron en esta investigación, señalaron la importancia de contar con sistemas informáticos intuitivos, amigables y de fácil navegación para conocer atractivos turísticos, hacer reservas, realizar compras y conocer e interactuar con personas en línea.

En el análisis de los resultados también se pudo determinar la preferencia que los turistas dan a los canales de comunicación a la hora de realizar consultas de servicios turísticos, incluyendo Kioscos digitales y, como se puede apreciar en el ranking que se muestra en la figura 2, Facebook es el líder en preferencias (64\%), seguido de Kiosko digital e Instagram (55\%) y las Plataformas Web (53\%), lo cual ubica estos mecanismos de interacción en una posición estratégica expectante para la industria turística.

En general, los turistas ratifican la relevancia que tiene para ellos la existencia de puntos de contacto accesibles. Como se aprecia también en la figura 2, las preferencias de los turistas evolucionan desde un 
94\% que menciona el Terminal Terrestre hasta un 74\% que mencionan la Ruta del cacao y la Ruta de la Fé, como las más valoradas para disponer de puntos de contacto.

Preferencias de contacto

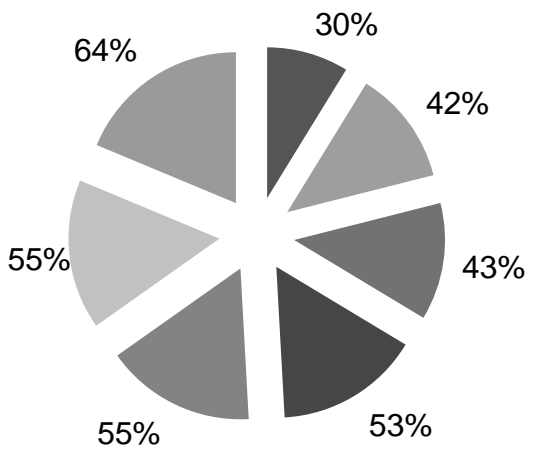

Ubicación de kioscos

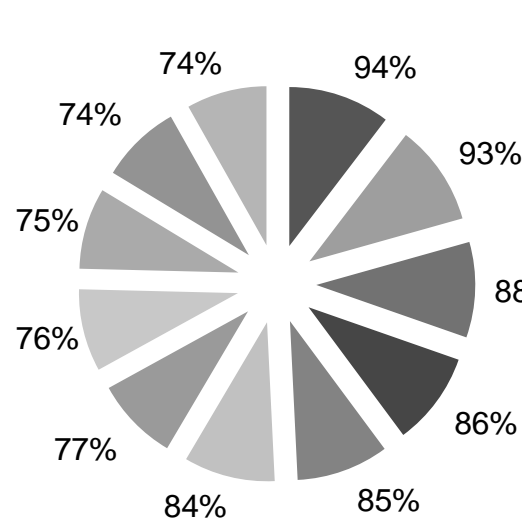

- Terminal Terrestre

- Aeropuerto

- Malecón 2000

- Comunidades con

atractivos turísticos

- Parque Histórico

Ruta del Sol

88\% — Ruta del Azúcar

Ruta del arroz

Ruta de la Aventura

Ruta de la fé

Ruta del cacao

Fig. 2: Preferencia de contacto y ubicación de kioscos

En cuanto a los niveles de satisfacción expresados por los turistas respecto de las tecnologías de la información y las comunicaciones implementadas en el sector, se aprecia que la valoración más alta la reciben los servicios tecnológicos amigables, accesibles y de fácil navegación $(78,39)$. De igual forma manifiestan satisfacción con los servicios en línea que ofrecen las agencias y tour operadores (76,56\%) acompañados de la capacidad de respuesta disponibles en las redes sociales $(75,78 \%)$. Por otra parte, los turistas valoran positivamente la información que se obtiene de los sistemas informáticos como plataformas web y App (74,22\%), especialmente cuando estas innovaciones se realizan en el sector turístico del Guayas (74,74\%) y que genera grados altos de satisfacción altos (72,92\%) al disponer de servicio en línea (24/7) intensivos y extensivo para la atención a turistas nacionales e internacionales.

\section{DISCUSIÓN}

En el estudio se identifican preferencias y experiencias positivas con plataformas turísticas como Trivago $(2,6 \%)$, Booking $(4,7 \%)$ que continúan ganando seguidores, sea por la seriedad en la gestión de los servicios que ofrecen a los proveedores turísticos, incluyendo a prestadores tales como SkyScanner $(1,6 \%)$ y Explore It (1\%). Complementariamente, se determinó que un 76,56\% de los encuestados manifiestan satisfacción con los servicios en línea que ofrecen las agencias y tour operadores, sea a través de sus sitios web y redes sociales, sin embargo, algunos visitantes aprecian que estos servicios les resultan escasamente operativos y con falencias en inmediatez de respuesta y confiabilidad. Los problemas aquí determinados, ya fueron analizados por Rodríguez-Vázquez et al. (2017) quien, basado en la herramienta online LikeAlyzer, pudo corroborar que no todos los usuarios utilizan redes sociales de igual modo ni con la misma efectividad en tiempos de respuesta, comercialización y promoción, lo cual sigue restando eficiencia a sus acciones.

Se observa así mismo que un $74,22 \%$ de los encuestados indica que la información que obtienen de los sistemas informáticos, plataformas web y App de la Provincia del Guayas es relevante, pertinente y precisa. Para otros más exigentes, la información aun no satisface todas sus expectativas, pues está muy sesgada o limitada a los mismos sitios y servicios que se han venido promocionando en los últimos años. Tales percepciones, estudiadas por Rodríguez-Vázquez et al., (2017), siguen presentado límites el uso de plataformas, limitando la interactividad, retroalimentación y escucha de los clientes como para que asignen valor a los negocios. En este contexto, un 25,26\% registra que la gestión tecnológica del sector turístico es regular y en muchos casos es catalogada como mala. También un $27,08 \%$ considera que el servicio en línea 24/7 es inoperativo e ineficiente en algunos horarios generando demoras en algunos procedimientos de turismo. Con relación a este último punto, Rivera y Símbala (2016) comentan que la empresa pública de turismo de Guayaquil debe activar acciones disruptivas cambiando la forma de realizar prestaciones en línea, si se quiere tener un cliente atendido con eficacia, que obtenga satisfacción y por ende, propicie conductas de fidelización y lealtad a dichos servicios.

Por otro lado, para un $75,78 \%$ de los entrevistados la atención recibida es buena o excelente, pero el resto aprecia algunas malas prácticas, que como ya ha sido estudiado (Assaker, et al., 2011), los turistas buscan 
acceder a mayor contenido útil que les genere confianza. A pesar de estas carencias, aproximadamente un $79 \%$ de los turistas coincidieron en que los servicios tecnológicos habilitados son, en cierta forma, amigables, accesibles y de fácil navegación. Prueba de ello son las nuevas plataformas de turismo en Quito, apreciada por un $72 \%$ de los turistas nacionales e internacionales, todo lo cual evidencia grados relevantes de segmentación (Sung, et al., 2015). En general, los hallazgos permiten atraer más turistas a la ciudad de Quito, a través de plataformas web calificadas de muy amigables y que permiten a los usuarios acceder a itinerarios y información de la ciudad, horarios, lugares de visita y agendas, entre otros.

Por último, un $94,70 \%$ de los turistas encuestados califican como importantes la apertura de canales de comunicación basados en tecnologías porque se accede a información turística de los agentes comercializadores y canales de comercialización publicitaria, pudiendo estos medios incidir directamente en la satisfacción y fidelización de los clientes como lo ratifica Tafur et al., (2018), indicando que se debe apuntar al rediseño radical de los procesos del negocio (Webster e Ivanov, 2014), sustentados en una tecnología que permita mejorar resultados en costes, calidad, servicio, y capacidad de respuesta como lo señala Rivera y Símbala (2016).

\section{CONCLUSIONES}

De acuerdo al trabajo presentado y a los resultados obtenidos se pueden arribar a las siguientes conclusiones principales:

1. La percepción que tienen los turistas nacionales e internacionales con relación a la integración tecnológica, centralización y autogestión de la información turística es de alta satisfacción apreciándose que las redes sociales y las aplicaciones móviles ganan adeptos como medios utilizados para la gestión de viajes turísticos, calificándolos como amigables, accesibles y de fácil navegación.

2. Por otro lado, Facebook, kioscos digitales, Instagram y las plataformas web son los canales de comunicación preferidos por los turistas, quienes valoran positivamente aquellos canales de comunicación basados en las nuevas tecnologías de la información y las comunicaciones para realizar búsquedas y dar tratamiento a la información turística de reservas, compras e interacción personalizada.

3. Los turistas valoran positivamente y se acepta en alto grado, la implantación en el territorio del Guayas los kioscos digitales turísticos de auto consulta y de autogestión en puntos estratégicos de la Provincia del Guayas por cuanto contribuyen a una mejor gestión de la información, las comunicaciones sectoriales del turismo y, por ende, incidir sobre la satisfacción y fidelización de los turistas que visitan el Guayas, Ecuador.

\section{REFERENCIAS}

Alegre, J. y Garau, J., The Factor Structure of Tourist Satisfaction at Sun and Sand Destinations. https://doi.org/10.1177/0047287509349270 Journal of Travel Research, 50(1), 78-86 (2011)

Assaker, G., Vinzi, V. E. y O'Connor, P., Examining the Effect of Novelty Seeking, Satisfaction, and Destination Image on Tourists' Return Pattern: A two Factor, Non-Linear Latent Growth Model. https://DOI:10.1016/j.tourman.2010.08.004 Tourism Management, 32(4), 890-901 (2011)

Castillo, E. A., Herrera, G. P. y Zambrano, D. I. Impacto Económico del Turismo en la Economía Del Ecuador. https://bit.ly/33FzqT8 Anais Brasileiros de Estudios Turísticos-ABET, 6(2), 69-81 (2016)

CGTI, Principales indicadores de turismo 2017-2018. https://bit.ly/3dgNpDo Coordinación General de Estadística e Investigación (2018)

CMVyT, Consejo Mundial de Viajes y Turismo. https://bit.ly/2xILInW Travel \& Tourism Economic Impact 2015 WORLD (2015)

Croes, R. R., Rivera, M.S., Ramirez, X.A., y Pizam, A., Plan Maestro de Desarrollo Turístico para la Provincia del Guayas. https://bit.ly/2UbKMv5 Consejo Provincial de la Provincia del Guayas \& La Cámara Provincial de Turismo del Guayas, Ecuador, 58-88 (2009)

Díaz, E. A. y Onofri, M. C., Microcréditos Solidarios: Un Enfoque de Reducción de Pobreza a Través del Turismo. http://oaji.net/articles/2017/3377-1517029390.pdf Transitare Vol. 3: 188-206 (2017)

ENEDyS., Encuesta Nacional de Empleo, Desempleo y Subempleo. https://bit.ly/2vLWwv2 Instituto Nacional de Estadística y Censos (2017)

Cuadrado, R. y Fernández, M.T. El Impacto de las Nuevas Tecnologías en el Sector Turístico: Aplicación de la Realidad Aumentada al Turismo Cultural. https://bit.ly/3acWg7p International Journal of World of Tourism, Vol. 1, №. 2 Págs. $10-$ 18 (2014) 
Freiberg, A., Stover, J.B. y de la Iglesia, G. Polychoric And Tetrachoric Correlations In Exploratory And Confirmatory Factorial Studies. Prensa Médica Latinoamericana - ISSN 1688-4094 Ciencias Psicológicas, VII (2): 151 - 164 (2013)

Gracia, G. E., y Torres, P. D., El Impacto de la Comunicación Pública en el Desarrollo del Turismo Interno en el Ecuador. https://bit.ly/2Ja9TYU Alternativas, 16(2), 42-66 (2015)

Gregory R.L. Eye and Brain, the Psycology of Seeing. $3^{\circ}$ edición: revised and updated, Mc Graw-Hill, New York, EEUU (2005)

Guillén, S. Vadece, un Modelo para la Gestión de Marketing, para Potenciar el Turismo Rural y Comunitario. https://doi.org/10.17979/redma.2016.01.017.4865 Redmarka, 1(17), 85-103 (2016)

Herington, C., Merrilees B. y Wilkins, H. Preferences for Destination Attributes: Differences Between Short and Long Breaks. https://doi.org/10.1177/1356766712463718 Journal of Vacation Marketing, 19(2), 149-163 (2013)

Hernández, R., Fernández, C. y Baptista, M. P. Metodología de la Investigación. Sexta Edición. Editorial Mc Graw Hill. México (2014)

Larrea, M.F. y Cevallos, A. Herramientas del Turismo 2.0 para la Promoción de la Ciudad de Guayaquil. https://doi.org/10.31095/podium.2016.30.6 Podium (30), 85-96 (2016)

Llorente-Bousquets, J. y Ocegueda, S. Estado del Conocimiento de la Biota https://bit.ly/2UdigJK en Capital Natural de México, vol. I: Conocimiento actual de la biodiversidad. Conabio, México, pp. 283-322 (2008)

Mendoza, E., Yumisaca, J., Rendón, M. F. y Ullauri, N. Observatorio Turístico: Una Herramienta de Gestión para el Turismo de Sol y Playa en la Provincia de Santa Elena. https://doi.org/10.26423/rctu.v3i3.210 Revista Científica y Tecnológica Upse, 3(3), 172-185 (2016)

OMT, Panorama Mundial del Turismo Internacional https://www.e-unwto.org/doi/pdf/10.18111/9789284418152 Organización Mundial del Turismo. (2016)

PLANDETUR, Plan Estratégico de Desarrollo de Turismo Sostenible para Ecuador 2020. https://bit.ly/3apdkXy

Reyes, M. V., Ortega, Á. F. y Machado, E. L. Integración y Sostenibilidad del Turismo Comunitario en Pastaza. Ecuador. http://retos.mes.edu.cu/index.php/retojs/article/view/167/156 Retos Turísticos, 14(2) (2015)

Rivera, C.Y. y Símbala, A.B. Propuesta de Optimización del uso de Herramientas Digitales, Utilizadas por la Empresa Pública de Turismo de Guayaquil para la Difusión del Cantón. https://bit.ly/3bjhYqd Tesis de Grado, Ingeniero en Administración de Empresas Turísticas y Hoteleras. Universidad Católica Santiago de Guayaquil, p.96 (2016)

Rodríguez-Vázquez, C., Rodríguez-Fernández, M. M., Sánchez-Fernández, M. D., Mahauad-Burneo, M. D. y MartínezFernández, V. A. Impacto de las Tecnologías en la Transformación del Modelo de Negocio de las Agencias de Viajes en Ecuador: del Ámbito Analógico al Digital. https://bit.ly/397YoMc Revista Espacios ISSN 0798 1015 38(59) (2017)

SNPyD, Plan nacional para el buen vivir 2013 - 2017 https://bit.ly/2QDYHI2 Secretaría Nacional de Planificación y Desarrollo. (2013)

Solomon Michael R. Comportamiento del consumidor. https://bit.ly/3acLeit Pearson Educación, Prentice Hall, Séptima Edición, México. (2008)

Song, H., Dwyer, L., Li, G. y Cao, Z. Tourism Economics Research: A Review and Assessment. https://DOI:10.1016/j.annals.2012.05.023 Annals of Tourism Research, 39(3), 1653-1682 (2012)

Sung, Y.K., Chang, K. C. y Sung, Y.F. Market Segmentation of International Tourists Based on Motivation to Travel: A Case Study of Taiwan. https://doi.org/10.1080/10941665.2015.1080175 Asia Pacific Journal of Tourism Research, 1-21 (2015)

Tafur, G.C., Vélez, C.I., Alejo, O.J., Zumba, R.M. y Jácome, J. Desarrollo Tecnológico del Sector Turístico en la Ciudad de Guayaquil (Ecuador). https://bit.ly/2xkwtLE Revista Espacios, ISSN 0798101539 (44) (2018)

Webster, C. y Ivanov, S.H. Transforming Competitiveness into Economic Benefits: Does Tourism Stimulate Economic Growth in More Competitive Destinations? http://DOI:10.1016/j.tourman.2013.06.003 Tourism Management, 40, 137-140 (2014)

WTM , Global Trends Report 2016 https://bit.ly/2y5qniZ World Travel Market in Association with Euromonitor International (2016). 